\title{
Sagittal spinal-pelvic alignment in patients with Crowe type IV developmental dysplasia of the hip
}

\author{
Peng Ren ${ }^{1,2+}$, Xiangpeng Kong ${ }^{2 \dagger}$, Wei Chai ${ }^{2^{*}}$ and Yan Wang ${ }^{2^{*}}$
}

\begin{abstract}
Background: The impact of high dislocated dysplastic hips on spinal-pelvic alignment has not been well described. This study aims to evaluate compensatory spinal radiographic changes and presence of back pain in patients with Crowe type IV developmental dysplasia of the hip (DDH).

Methods: An observational study was conducted from July 2016 to December 2017, and 49 consecutive patients with Crowe IV DDH were enrolled. Forty-nine sex- and age-matched asymptomatic healthy adults were recruited as the controls. The sacral slope (SS), lumbar lordosis (LL), spino-sacral angle (SSA), C7 tilt (C7T), and sagittal vertical axis (SVA [C7]) were measured on lateral whole spine radiographs. The presence of low back pain and visual analogue scale (VAS) scores were recorded.

Results: The patients with Crowe IV DDH showed significantly greater SS $\left(47.5 \pm 7.5^{\circ}\right.$ vs. $\left.40.4 \pm 6.7^{\circ}, p<0.05\right), \mathrm{LL}$ $\left(-63.7 \pm 9.2^{\circ}\right.$ vs. $\left.-53.3 \pm 11.5^{\circ}, P<0.05\right)$, SSA $\left(141.8^{\circ} \pm 7.2^{\circ}\right.$ vs. $\left.130.6 \pm 7.9^{\circ}, p<0.05\right)$, C7T $\left(93.9 \pm 3.6^{\circ}\right.$ vs. $91.1 \pm 3.7^{\circ}$, $P<0.05)$, and lower SVA(C7) $(-16 \mathrm{~mm}[-95-45]$ vs. $6.4 \mathrm{~mm}[-52-47], p<0.05)$ compared to the controls. The patients with bilateral Crowe IV DDH also exhibited larger SS, LL, SSA, and C7T and a smaller SVA (C7) than those with unilateral Crowe IV DDH. Sixty-three percent of the patients with Crowe IV DDH reported low back pain.

Conclusion: The patients with Crowe IV DDH exhibited abnormal spinal-pelvic alignment characterized by anterior pelvic tilt, lumbar hyperlordosis, and a backward-leaning trunk. Bilateral Crowe IV DDH had a greater impact on spinal-pelvic alignment than unilateral Crowe IV DDH.
\end{abstract}

Keywords: Spinal-pelvic alignment, Sacral slope, Lumbar lordosis, Developmental dysplasia of the hip, Hip-spine syndrome

\section{Background}

The compensatory mechanisms of the spine, pelvis, and lower limbs are essential in daily activities to maintain a stable, upright posture in the sagittal plane [1]. Sagittal spinal-pelvic alignment, first described in 1998, has been well studied in patients with spinal disorders, including low back pain, spondylolisthesis, and spinal deformities

\footnotetext{
*Correspondence: chaiwei301@163.com; wangyanspinejoint@163.com

${ }^{+}$Peng Ren and Xiangpeng Kong are co-first author

${ }^{2}$ Department of Orthopaedics, the First Medical Center, Chinese PLA General Hospital, 28 Fuxing Road, Beijing 100853, China

Full list of author information is available at the end of the article
}

[2-5]. Patients with severe hip osteoarthritis (OA) have also been reported to have abnormal sagittal spinalpelvic alignment $[6,7]$.

The incidence of hip dysplastic dislocation was reported to be between $0.1-0.15 \%$ in newborns and often involved unilateral side [8]. Girls are more likely to be involved than boys $[9,10]$. In adults, high dislocated dysplastic hips are classified as Crowe IV developmental dysplasia of the hip (DDH), and may lead to hip pain, limp or wobbling gait [11]. Matsuyama reported changes in the sagittal alignment of the spine in patients with

(c) The Author(s). 2020 Open Access This article is licensed under a Creative Commons Attribution 4.0 International License, which permits use, sharing, adaptation, distribution and reproduction in any medium or format, as long as you give appropriate credit to the original author(s) and the source, provide a link to the Creative Commons licence, and indicate if changes were made. The images or other third party material in this article are included in the article's Creative Commons licence, unless indicated otherwise in a credit line to the material. If material is not included in the article's Creative Commons licence and your intended use is not permitted by statutory regulation or exceeds the permitted use, you will need to obtain permission directly from the copyright holder. To view a copy of this licence, visit http://creativecommons.org/licenses/by/4.0/ The Creative Commons Public Domain Dedication waiver (http://creativecommons.org/publicdomain/zero/1.0/) applies to the data made available in this article, unless otherwise stated in a credit line to the data. 
bilateral Crowe IV DDH [12]. The author observed that total sagittal alignment of the spine in these patients was compensated for by anterior tilt of the pelvis and lumbar hyperlordosis. In this regard, the main clinical symptom was lower back pain instead of leg pain [12]. To our knowledge, this is the only study that has focused on sagittal spinal alignment in these particular cases. However, the parameter representing pelvic tilt in his study was sacrum inclination (SI) angle, which was seldom reported in current literature. In addition, the patients with unilateral Crowe IV DDH were not included.

Thus, the observational study was conducted to evaluate compensatory spinal radiographic changes and presence of back pain in patients with Crowe IV DDH.

\section{Methods}

This observational study was conducted from July 2016 to December 2017 with approval by the ethics committee at our institution, and informed consent was obtained from all subjects. Patients suffering from Crowe IV DDH who underwent total hip arthroplasty in our hospital were recruited. The exclusion criteria included the following: (1) a prior history of spinal surgery; (2) marked osteoarthritis in the knee or ankle; (3) lower limb radicular pain; (4) symptomatic spinal stenosis; (5) neurological disorders affecting postural control; (6) other diseases that would affect spinal-pelvic alignment, including lumbar disc herniation, spondylolisthesis, primary spinal deformity, severe lumbar spine degenerative change (Weiner Grade 3, which was performed by a senior surgeon reviewing the images and grading them according to the Weiner system.) [13], and a history of thoracolumbar fracture; and (7) patient preference by declining to participate in the study. During the study period, 52 patients were assessed and 49 patients who met the criteria were recruited consecutively into the study. Three patients were excluded, including one due to thoracolumbar kyphosis of $26^{\circ}$, one with a rigid scoliosis of $35^{\circ}$ that involved the entire thoracic and lumbar spines, and one with L4/L5 and L5/S1 lumbar disc herniation. Based on the hip involved, patients were divided into two groups: patients with unilateral Crowe IV DDH $(n=39)$, and patients with bilateral Crowe IV DDH $(n=10)$.

Once enrolled, patients were asked about the presence and duration of chronic low back pain (LBP). Chronic LBP was defined as consecutive pain for greater than 3 months in the lumbar spine. The visual analogue scale (VAS) was used to assess pain intensity. Other frequentlyused methods, such as the Oswestry Disability Index (ODI) and Roland-Morris Disability Questionnaire (RMDQ), were not utilized due to hip's confounding effect. Forty-nine age (less than 2 years) and gender (oneto-one correspondence) matched healthy volunteers who fulfilled the following criteria were recruited as controls:
(1) no pathology or surgical history of the pelvis, hip, or lower limbs; (2) no spinal trauma, deformity, or surgical history; (3) no spinal neurologic symptoms; (4) no severe lumbar spine degenerative change (Weiner Grade 3).

\section{Radiography and measurements}

Standing lateral and anteroposterior full spine radiographs and oblique lumbar radiographs were obtained [14]. For the lateral view, patients stood in a natural, erect posture with knees in extension, and relaxed upper limbs with elbows half bent and hands resting on a support. For the anteroposterior view, patients stood in the same manner as the lateral view, with the exception being that the upper limbs were hanging alongside the body.

The parameters of the sagittal spine-pelvis, including sacral slope (SS), lumbar lordosis (LL), spino-sacral angle (SSA), C7 tilt (C7T), and sagittal vertical axis (SVA [C7]) were measured by two independent observers on the lateral radiographs using the picture archiving and communication system (PACS) to assess inter-observer reliability. After 4 weeks, these measurements were repeated to assess intra-observer reliability. The methods used to measure these parameters are as follows (Figs. 1 and 2):

1. SS [2]: the angle between the sacral endplate and a horizontal line.

2. LL: the angle between the superior endplate of L1 and the S1 endplate.

3. SSA [15]: the angle between the sacral endplate and a line from the center of the $\mathrm{C} 7$ vertebral body to the center of the sacral endplate.

4. C7T [15]: the angle between the horizontal line and a line from the center of the $\mathrm{C} 7$ vertebral body to the center of the sacral endplate.

5. Sagittal vertical axis (SVA(C7)) [16]: the horizontal distance from the posterior edge of the sacral endplate to the plumb line passing through the center of the $\mathrm{C} 7$ vertebral body (C7PL).

Because pelvic incidence (PI) and pelvic tilt (PT) were the parameters relying on the normal relationship between femoral head and acetabulum, measuring PT and PI in Crowe IV DDH patients were inaccurate [2]. We didn't include PT and PI in our study .

\section{Statistical analysis}

All statistical analyses were performed by SPSS version 22 (IBM, Chicago, IL). Measurement data was expressed as the mean and standard deviation. Measurement data were analyzed by independent sample $t$ test or analysis of variance. Categorical data was analyzed by the chisquare test. The intraclass correlation coeffificient (ICC) was used to determine the variations of the different measurements: 0.81 to 1.00 was regarded as nearly 


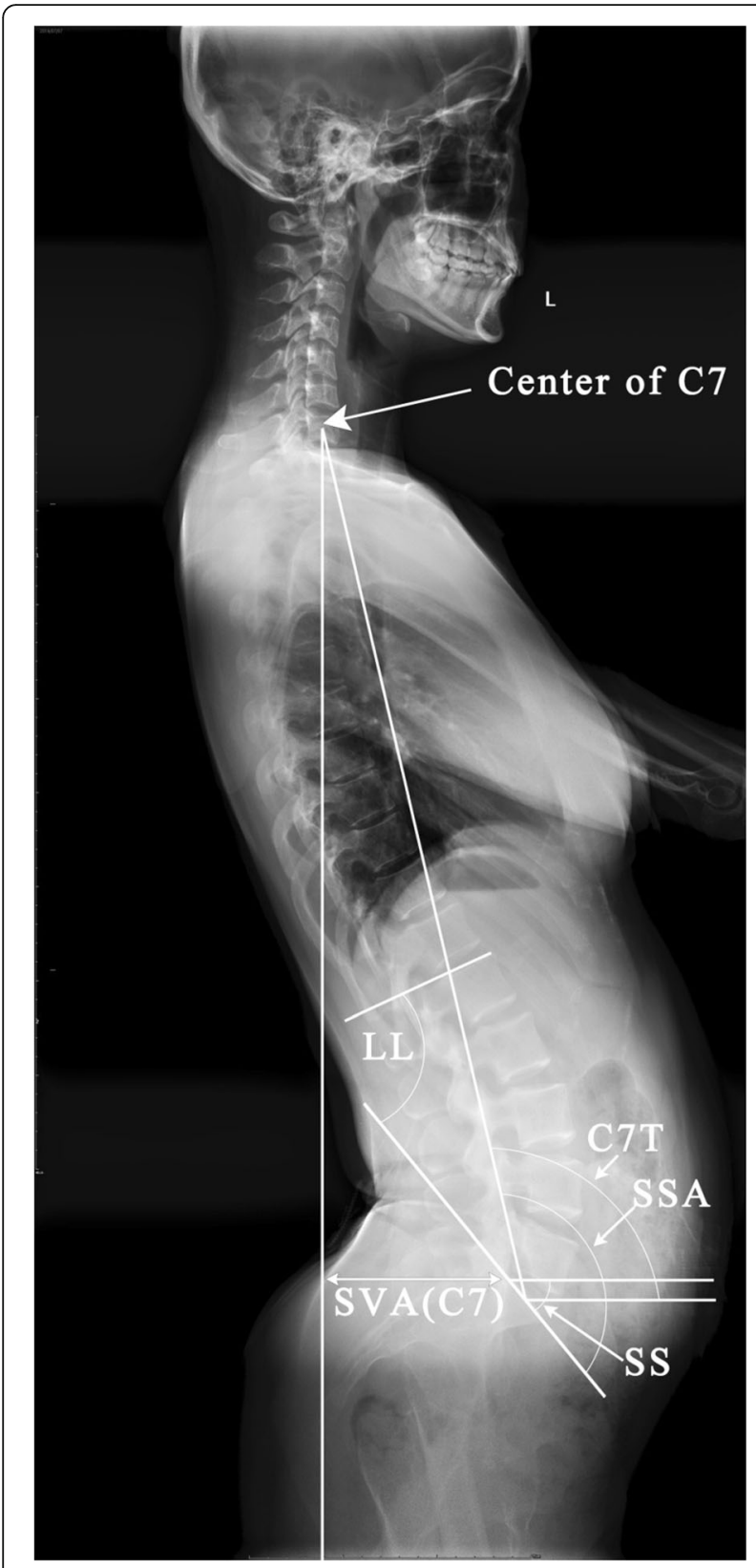

Fig. 1 Illustration of the radiographic parameters of the spinal-pelvic alignment in bilateralhigh dislocated dysplastic hips. This patient has an anteriorly inclined pelvis, lumbar hyperlordosis, and a backwardleaning trunk. SS sacral slope, LL lumbar lordosis, C7T C7tilt, SSA spino-sacral angle, SVA(C7) sagittal vertical axis(C7)

perfect reliability; 0.61 to 0.80 , strong reliability; 0.41 to 0.60 , moderate; 0.21 to 0.40 , fair; and 0 to 0.20 , poor. A $p$-value $<0.05$ was considered significant.

\section{Results}

General demographic data of the patients and controls are summarized in Table 1 . There were 5 males and 44 females, and the average age was $39.9 \pm 9.8$ years (range 22-67 years). There were 39 patients ( 4 males and 35

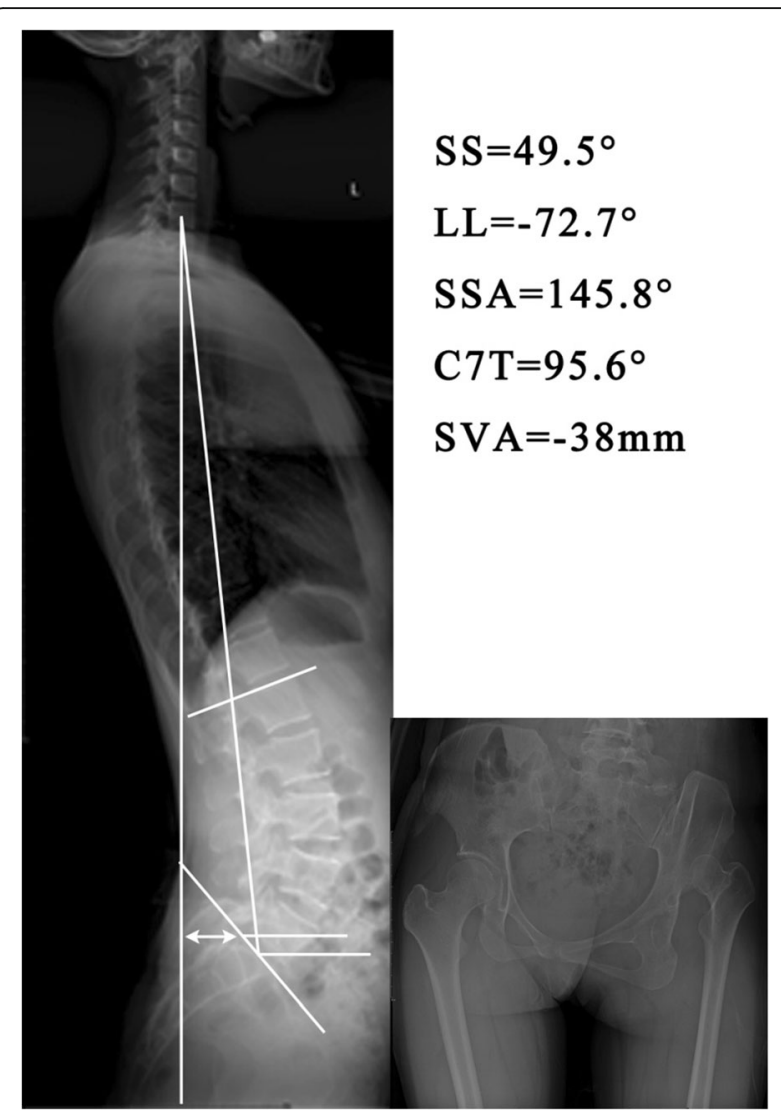

Fig. 2 The sagittal spinal-pelvis alignment of a patient with unilateral hip high dislocated dysplasia. This patient had a significantly anterior tilted pelvis, lumbar hyperlordosis, and a backward-leaning trunk

females with an average age $40.1 \pm 8.9$ years [range 22 62 years]) with unilateral Crowe IV DDH, and 10 patients (10 females with an average age $39.2 \pm 13.4$ years [range 23-67 years]) with bilateral Crowe IV DDH. There were 5 males and 44 females with an average age $39.9 \pm 6.7$ years (range $25-62$ years) in the control group. The age and gender distributions between the groups revealed no significant differences.

The mean values and standard deviations of the radiographic parameters are showed in Table 2.

Compared to asymptomatic controls, the patients with Crowe IV DDH showed significantly greater SS, LL, SSA, C7T, and lower SVA(C7). Among Crowe IV DDH patients, the bilateral had a significantly greater SS, LL, SSA, and C7T and lower SVA(C7) than the unilateral.

Reliability analysis demonstrated that the ICC of both inter-observer agreements and intra-observer agreements were larger than 0.81 .

Thirty-one (63.2\%) patients with Crowe IV DDH reported LBP, including 24 patients with unilateral Crowe IV DDH and 7 with bilateral Crowe IV DDH. Among the patients with LBP, the unilateral and bilateral Crowe IV DDH patients had an average VAS of $5.3 \pm 1.6$ and 
Table 1 Patient demographics

\begin{tabular}{|c|c|c|c|}
\hline & $\begin{array}{l}\text { Unilateral Crowe IV } \\
\mathrm{DDH}(n=39)\end{array}$ & $\begin{array}{l}\text { Bilateral Crowe IV } \\
\text { DDH }(n=10)\end{array}$ & $\begin{array}{l}\text { Control } \\
(n=49)\end{array}$ \\
\hline \multicolumn{4}{|l|}{ Age } \\
\hline Mean \pm SD & $40.1 \pm 8.9$ & $39.2 \pm 13.3$ & $39.9 \pm 7.8$ \\
\hline Range & $22-62$ & $23-67$ & $20-57$ \\
\hline \multicolumn{4}{|l|}{ Gender } \\
\hline Male & $4(10.3 \%)$ & $0(0)$ & $4(8.2 \%)$ \\
\hline Female & 35 (90.7\%) & $10(100 \%)$ & $45(91.8 \%)$ \\
\hline BMI $\left(\mathrm{kg} / \mathrm{m}^{2}\right)$ & $22.6 \pm 4.0$ & $21.2 \pm 3.0$ & $22.8 \pm 3.2$ \\
\hline Height & $158.4 \pm 6.9$ & $157.4 \pm 6.0$ & $160 \pm 6.3$ \\
\hline weight & $56 \pm 11.2$ & $53 \pm 8.6$ & $58 \pm 10.7$ \\
\hline \multicolumn{4}{|l|}{ Dislocated hip } \\
\hline Left & 18 & 10 & - \\
\hline Right & 21 & 10 & - \\
\hline \multicolumn{4}{|c|}{ Non-dislocated hip } \\
\hline Healthy & 29 & - & - \\
\hline Crowe type I & 2 & - & - \\
\hline Crowe type II & 4 & - & - \\
\hline Crowe type III & 4 & - & - \\
\hline
\end{tabular}

$5.8 \pm 1.7$, respectively. There were no differences in prevalence or VAS between unilateral and bilateral Crowe IV DDH group.

\section{Discussion}

In this study, we found that the patients with Crowe IV DDH showed significantly greater SS, LL, SSA, C7T, and lower SVA(C7) than controls, which was more obvious in the patients with bilateral Crowe IV DDH. Thirty-one (63.2\%) Crowe IV DDH patients reported LBP. However, there were no significant differences in prevalence or
VAS between unilateral and bilateral Crowe IV DDH group.

Several studies reported the impact of hip disease on spine-pelvic alignments, and their results were inconsistent. In a prospective study, Weng reported a significantly larger SS and smaller PT in patients with hip OA [6]. Piazzolla reported that patients with hip OA and low back pain have significantly increased SS, LL, and forward inclination of the trunk [17]. Yoshimoto chose individuals with low back pain as controls and reported that patients with hip OA showed significantly higher PI, SS and LL [7]. However, Sariali found that patients with hip OA had significantly lower SS than asymptomatic healthy controls [18]. In patients with secondary OA with hip dysplasia, Okuda found that pelvic inclination tended to increase in pre/early-stage OA patients. With aging, patients with OA maintained the lumbar lordotic angle and did not develop a posterior sacral slope angle [19]. Our results showed the patients with Crowe IV DDH have significantly increased SS, LL.

Crowe IV DDH may represent one of the most severe conditions among the spectrum of hip pathology. One previous study found that total sagittal alignment of the spine in patients with bilateral hip dislocations was compensated for by anterior tilt of the pelvis and lumbar hyperlordosis [12]. However, the parameter SI, which was used to evaluate sacral rotation, was not frequently reported in the recent literature.

The present study used SS to evaluate pelvic rotation. Our results revealed that the patients with Crowe IV $\mathrm{DDH}$ had significantly larger SS than that of controls. As a parameter closely correlated with SS, the LL angle showed the similar results. Our results also indicated that even with unilateral dislocated hip, the spinal-pelvic alignment can be altered. However, with one nondislocated hip sustaining the trunk, the pelvic

Table 2 Comparisons of the sagittal spinal-pelvic alignment parameters and LBP

\begin{tabular}{|c|c|c|c|c|}
\hline & \multirow{2}{*}{$\begin{array}{l}\text { Control } \\
(n=49)\end{array}$} & \multicolumn{3}{|l|}{ Crow IV DDH } \\
\hline & & Total $(n=49)$ & Unilateral $(n=39)$ & Bilateral $(n=10)$ \\
\hline $\mathrm{SS}\left({ }^{\circ}\right)$ & $40.4 \pm 6.7$ & $47.5 \pm 7.5^{*}$ & $45.7 \pm 7.2^{*}$ & $54.3 \pm 2.8^{* \boldsymbol{\Lambda}}$ \\
\hline $\mathrm{LL}\left({ }^{\circ}\right)$ & $-53.3 \pm 11.5$ & $-63.7 \pm 9.2^{*}$ & $-61.3 \pm 8.8^{*}$ & $-72.9 \pm 3.0^{*} \boldsymbol{\Lambda}$ \\
\hline $\operatorname{SSA}\left({ }^{\circ}\right)$ & $130.6 \pm 7.9$ & $141.8 \pm 7.2^{*}$ & $139.7 \pm 6.3^{*}$ & $150.2 \pm 3.6^{* \boldsymbol{\Lambda}}$ \\
\hline $\mathrm{C} 7 \mathrm{~T}\left({ }^{\circ}\right)$ & $91.1 \pm 3.7$ & $93.9 \pm 3.6^{*}$ & $93.3 \pm 3.6^{*}$ & $96.3 \pm 2.3^{* \boldsymbol{\Lambda}}$ \\
\hline SVA $(\mathrm{mm})$ & $6.4(-52-47)$ & $-16(-95-45) *$ & $-11.1(-70-44.5) *$ & $-32.6(-95-0) * \boldsymbol{\Delta}$ \\
\hline Number of patients with LBP & & $31(63.2 \%)$ & $24(61.5 \%)$ & $7(70 \%)$ \\
\hline Spine VAS scores of patients with LBP & & & $5.3 \pm 1.6$ & $5.8 \pm 1.7$ \\
\hline
\end{tabular}

Comparisons between Crow IV DDH patients and controls (independent sample t test). ${ }^{*} p<0.05$

Comparisons between unilateral and bilateral Crowe IV DDH group. (independent sample t test). ${ }^{\wedge} p<0.05$

There were no differences in prevalence $(p=0.13)$ or low back pain intensity based on VAS $(p=0.48)$ between patients with low back pain in unilateral and bilateral Crowe IV DDH group

$D D H$ developmental dysplasia of the hip, LBP low back pain, VAS visual analogue scale 
orientation in unilateral cases may not change as much as bilateral cases.

SVA(C7) was measured in the present study to assess the global spinal balance. The spine is considered slightly unbalanced if C7PL located between the femoral head and the posterior edge of the sacral plate, and severely unbalanced if it is located anterior to the femoral heads. Our results revealed the patients with Crowe IV DDH had an average lower SVA than control group. This means the patients with Crowe IV DDH present with a tendency toward a backward-leaning trunk, which was in accordance with the description of the relationship between femoral head, central gravity line, and C7PL [20].

Other parameters related to $\mathrm{C} 7$ were $\mathrm{C} 7 \mathrm{~T}$ and SSA. $\mathrm{C} 7 \mathrm{~T}$ is a functional parameter that reflects the global orientation of spine. SSA is a parameter that quantifies the global kyphosis of the entire spine and pelvis. C7T, SSA and SS are linked by the following equation: SSA = C7T + SS [16]. In the present study, the average SSA and C7T in the patients with Crowe IV DDH were significantly larger than those in the control group. This result also indicated that patients with Crowe IV DDH present with a more backward global orientation of the spine, and besides, a decreased global kyphosis of the entire spine-pelvis.

Hip diseases would lead to the change of sagittal spinal-pelvic alignment, while different diseases may have different mechanisms for this change. Hip flexion contracture has been reported as one cause of pelvic anteversion and lumbar hyperlordosis in patients with severe hip OA [21]. However, severe dysplastic hips often reveal a greater range of motion (ROM) than healthy hips. Therefore, spinal alignment changes in Crowe IV DDH cannot be attributed to hip flexion contracture. In the Crowe IV DDH patients, the femoral head is dislocated upward and backward, and the trunk supporting function of hip joint loses. Meanwhile, anatomical course of soft tissue also changed. It is the two factors that make the pelvic rotation anteriorly and thereby increase SS. Lumbar lordosis will then be increased to compensate for the anterior pelvic tilt and to meet the criterion of central gravity line passing approximately through the femoral head.

LBP caused by hip disease was termed "secondary" Hip-Spine Syndrome (HSS) by Offierski and MacNab [21]. They suggested that hyperlordosis of the lumbar spine may result in abnormal force on the posterior facets and LBP. Parvizi reported that $49 \%$ of patients with end-stage hip arthritis had LBP [22]. In the patients with Crowe IV DDH, the current authors considered that several factors may contribute to LBP, the first factor was the overloaded shear and compressing stresses on the posterior facet joints; the second factor was the asymmetric load distribution on posterior facet joints; the third factor was the fatigue of back muscles. Therefore, although the average age of the Crowe IV DDH patients in our study was only 40 years old, the observed LBP prevalence was $63.2 \%$.

There are several limitations in the present study. Firstly, several parameters related to hip centers were not measured, including PI and PT. Measuring PT and PI in the Crowe IVDDH patients were inaccurate due to the high dislocated hip joints. Secondly, though ages in our groups varied quite a bit, and the status of the contralateral hip differed in patients with unilateral hip dislocation, we did not set subgroups because of the small sample size. Thirdly, we did not attempt to explore the correlation between lumbago and the spinal-pelvic alignment. LBP can be caused by multiple factors, which would be taken into account in future research.

\section{Conclusion}

The patients with Crowe IV DDH exhibited abnormal spinal-pelvic alignment characterized by anterior pelvic tilt, lumbar hyperlordosis, and a backward-leaning trunk. Bilateral Crowe IV DDH had a greater impact on spinalpelvic alignment than unilateral Crowe IV DDH.

\section{Abbreviations \\ DDH: Developmental dysplasia of the hip; THA: Total hip arthroplasty; SS: sacral slope; LL: Lumbar lordosis; SSA: Spino-sacral angle; C7T: C7 tilt; SVA: Sagittal vertical axis; PT: Pelvic tilt; PI: Pelvic incidence; SI: Sacrum inclination; OA: Osteoarthritis; LBP: Low back pain; VAS: Visual analogue scale; ODI: Oswestry disability index; RMDQ: Roland-Morris disability questionnaire; C7PL: C7 plumbline; PACS: Picture archiving and communication system; ROM: Range of motion; HSS: Hip-Spine Syndrome; ICC: Intraclass correlation coeffificient}

\section{Acknowledgements}

Not applicable.

\section{Authors' contributions}

PR, XPK gathered and analyzed the data, and wrote the manuscript. WC, YW planed and supervised the study and revised the manuscript. All authors read and approved the final manuscript.

Funding

The authors declare that they have no funding.

Availability of data and materials

The datasets analysed during the current study are available from the corresponding author on reasonable request.

Ethics approval and consent to participate

This study has been approved by Institutional Ethics Committee of Chinese PLA General Hospital, and written informed consent was obtained from all subjects.

Consent for publication

Not applicable.

Competing interests

Not applicable. 


\section{Author details}

'Medical School of Chinese PLA, 28 Fuxing Road, Beijing 100853, China.

${ }^{2}$ Department of Orthopaedics, the First Medical Center, Chinese PLA General Hospital, 28 Fuxing Road, Beijing 100853, China.

\section{Received: 27 March 2020 Accepted: 13 October 2020}

\section{Published online: 17 October 2020}

\section{References}

1. Le Huec JC, Saddiki R, Franke J, et al. Equilibrium of the human body and the gravity line: the basics. Eur Spine J. 2011;20(Suppl5):558-63.

2. Legaye J, Duval-Beaupere G, Hecquet J, et al. Pelvic incidence: a fundamental pelvic parameter for three-dimensional regulation of spinal sagittal curves. Eur Spine J. 1998;7:99-103.

3. Chaleat-Valayer E, Mac-Thiong JM, Paquet J. Sagittal spino-pelvic alignment in chronic low back pain. Eur Spine J. 2011;20(Suppl 5):634-40.

4. Funao $\mathrm{H}$, Tsuji $\mathrm{T}$, Hosogane $\mathrm{N}$, et al. Comparative study of spinopelvic sagittal alignment between patients with and without degenerative spondylolisthesis. Eur Spine J. 2012;21:2181-7.

5. Zhu F, Bao H, He S, et al. Lumbo-femoral angle: a novel sagittal parameter related to quality of life in patients with adult scoliosis. Eur Spine J. 2015; 24(6):1244-50.

6. Weng WJ, Wang WJ, Wu MD, et al. Characteristics of sagittal spine-pelvisleg alignment in patients with severe hip osteoarthritis. Eur Spine J. 2014;24: 1228-36.

7. Yoshimoto H, Sato S, Masuda T, et al. Spinopelvic alignment in patients with osteoarthrosis of the hip: a radiographic comparison to patients with low back pain. Spine (Phila Pa 1976). 2005;30:1650-7.

8. Callaghan JJ, Rosenberg AG, Rubash HE. The Adult Hip. 2nd ed. 530 Walnut Street, Philadelphia, PA 19106 USA: Lippincott Williams \& Wilkins; 2007. p. 423.

9. Weinstein SL. Natural history of congenital hip dislocation (CDH) and hip dysplasia. Clin Orthop Relat Res. 1987:(225):62-76.

10. Dezateux C, Rosendahl K. Developmental dysplasia of the hip. Lancet. 2007; 369(9572):1541-52.

11. Crowe JF, Mani VJ, Ranawat CS. Total hip replacement in congenital dislocation and dysplasia of the hip. J Bone Joint Surg Am. 1979;61:15-23.

12. Matsuyama $Y$, Hasegawa $Y$, Yoshihara $H$, et al. Hip-spine syndrome: total sagittal alignment of the spine and clinical symptoms in patients with bilateral congenital hip dislocation. Spine (Phila Pa 1976). 2004;29:2432-7.

13. Weiner DK, Distell B, Studenski S, Martinez S, Lomasney L, Bongiorni D. Does radiographic osteoarthritis correlate with flexibility of the lumbar spine? J Am Geriatr Soc. 1994;42(3):257-63.

14. Morvan G, Mathieu P, Vuillemin V, et al. Standardized way for imaging of the sagittal spinal balance. Eur Spine J. 2011;20(Suppl 5):602-8.

15. Mac-Thiong JM, Roussouly P, Berthonnaud E, et al. Sagittal parameters of global spinal balance: normative values from a prospective cohort of seven hundred nine Caucasian asymptomatic adults. Spine (Phila Pa 1976). 2010; 35:E1193-8.

16. Roussouly P, Pinheiro-Franco JL. Biomechanical analysis of the spino-pelvic organization and adaptation in pathology. Eur Spine J. 2011;20(Suppl 5): 609-18.

17. Piazzolla A, Solarino G, Bizzoca D. Spinopelvic parameter changes and low back pain improvement due to femoral neck anteversion in patients with severe unilateral primary hip osteoarthritis undergoing total hip replacement. Eur Spine J. 2018;27(1):125-34

18. Sariali E, Lazennec JY, Khiami F, et al. Modification of pelvic orientation after total hip replacement in primary osteoarthritis. Hip Int. 2009;19:257-63.

19. Okuda T, Fujita T, Kaneuji A, et al. Stage-specific sagittal spinopelvic alignment changes in osteoarthritis of the hip secondary to developmental hip dysplasia. Spine (Phila Pa 1976). 2007;32:E816-9.

20. Hasegawa K, Okamoto M, Hatsushikano S. Standing sagittal alignment of the whole axial skeleton with reference to the gravity line in humans. J Anat. 2017;230:619-30

21. Offierski CM, MacNab I. Hip-spine syndrome. Spine (Phila Pa 1976). 1983;8: 316-21.

22. Parvizi J, Pour AE, Hillibrand A, et al. Back pain and total hip arthroplasty: a prospective natural history study. Clin Orthop Relat Res. 2010;468:1325-30.

\section{Publisher's Note}

Springer Nature remains neutral with regard to jurisdictional claims in published maps and institutional affiliations.

Ready to submit your research? Choose BMC and benefit from:

- fast, convenient online submission

- thorough peer review by experienced researchers in your field

- rapid publication on acceptance

- support for research data, including large and complex data types

- gold Open Access which fosters wider collaboration and increased citations

- maximum visibility for your research: over $100 \mathrm{M}$ website views per year

At BMC, research is always in progress.

Learn more biomedcentral.com/submissions 